\title{
ARTICLE
}

\section{Ambulatory blood pressure measurements are related to albumin excretion and are predictive for risk of microalbuminuria in young people with type 1 diabetes}

\author{
M. L. Marcovecchio • R. N. Dalton • C. P. Schwarze • \\ A. T. Prevost • H. A. W. Neil • C. L. Acerini • \\ T. Barrett • J. D. Cooper $\cdot$ J. Edge $\cdot$ J. Shield . \\ B. Widmer $\cdot$ J. A. Todd $\cdot$ D. B. Dunger
}

Received: 13 December 2008 / Accepted: 18 February 2009/Published online: 21 March 2009

(C) Springer-Verlag 2009

\begin{abstract}
Aims/hypothesis The relationship between BP and microalbuminuria in young people with type 1 diabetes is not completely clear. As microalbuminuria is preceded by a gradual rise in albumin excretion within the normal range, we hypothesised that ambulatory $\mathrm{BP}(\mathrm{ABP})$ may be closely related to albumin excretion and progression to microalbuminuria.
\end{abstract}

Electronic supplementary material The online version of this article (doi:10.1007/s00125-009-1327-6) contains supplementary material, which is available to authorised users.

M. L. Marcovecchio • C. P. Schwarze · C. L. Acerini •

B. Widmer $\cdot$ D. B. Dunger $(\triangle)$

Department of Paediatrics, University of Cambridge,

Level 8, Box 116, Addenbrooke's Hospital, Hills Road,

Cambridge CB2 0QQ, UK

e-mail: dbd25@cam.ac.uk

R. N. Dalton

WellChild Laboratory, King's College London,

Evelina Children's Hospital,

London, UK

\section{A. T. Prevost \\ Department of Public Health and Primary Care, \\ University of Cambridge,}

Cambridge, UK

H. A. W. Neil

Oxford Centre for Diabetes, Endocrinology and Metabolism,

University of Oxford,

Oxford, UK

\section{L. Acerini - D. B. Dunger}

Institute of Metabolic Science, University of Cambridge,

Addenbrooke's Hospital,

Cambridge, UK
Methods ABP monitoring (ABPM) was performed in 509 young people with type 1 diabetes (age median [range]: 15.7 [10.7-22.6] years) followed with annual assessments of three early morning urinary albumin:creatinine ratios (ACRs) and $\mathrm{HbA}_{1 \mathrm{c}}$. Systolic BP (SBP) and diastolic BP (DBP) and the nocturnal fall in BP were analysed in relation to ACR.

\section{T. Barrett}

Department of Paediatrics, Birmingham Children's Hospital, Birmingham, UK

\section{J. D. Cooper · J. A. Todd}

Juvenile Diabetes Research Foundation/Wellcome Trust Diabetes and Inflammation Laboratory, Department of Medical Genetics, Cambridge Institute for Medical Research,

University of Cambridge,

Cambridge, UK

\section{J. Edge}

Department of Paediatric Endocrinology and Diabetes,

Oxford Children's Hospital,

Headington,

Oxford, UK

J. Shield

Institute of Child Life and Health, UBHT Education Centre,

Bristol, UK 
Results All ABPM variables were significantly related to baseline $\log _{10}$ ACR $(p<0.001)$. After the ABPM evaluation, 287 patients were followed for a median of $2.2(1.0-5.5)$ years. ABP at baseline was independently related to mean ACR during follow-up. Nineteen initially normoalbuminuric patients developed microalbuminuria after 2.0 (0.2-4.0) years and their baseline daytime DBP was higher than in normoalbuminuric patients $(p<0.001)$. After adjusting for baseline $\mathrm{ACR}$ and $\mathrm{HbA}_{1 \mathrm{c}}$, there was an $11 \%$ increased risk of microalbuminuria for each $1 \mathrm{mmHg}$ increase in daytime DBP. Forty-eight per cent of patients were non-dippers for SBP and $60 \%$ for DBP; however, ACR was not different between dippers and non-dippers and there were no differences in the nocturnal fall in BP between normoalbuminuric and future microalbuminuric patients.

Conclusions/interpretation In this cohort of young people with type 1 diabetes, ABP was significantly related to ACR, and daytime DBP was independently associated with progression to microalbuminuria. Increasing albumin excretion, even in the normal range, may be associated with parallel rises in BP.

Keywords Albumin excretion - Ambulatory blood pressure monitoring · Blood pressure · Diabetic nephropathy .

Microalbuminuria . Type 1 diabetes $\cdot$ Young people

$\begin{array}{ll}\text { Abbreviations } \\ \text { ABP } & \text { Ambulatory BP } \\ \text { ABPM } & \text { Ambulatory BP monitoring } \\ \text { ACR } & \text { Albumin:creatinine ratio } \\ \text { DBP } & \text { Diastolic BP } \\ \text { HR } & \text { Heart rate } \\ \text { NFS } & \text { Nephropathy Family Study } \\ \text { SBP } & \text { Systolic BP } \\ \text { SDS } & \text { Standard deviation score }\end{array}$

\section{Introduction}

In patients with type 1 diabetes, microalbuminuria represents an important predictor for diabetic nephropathy and cardiovascular disease $[1,2]$. Increased arterial BP and the development of microalbuminuria are closely linked [1] and a familial predisposition to hypertension has been associated with the development of microalbuminuria [3]. Furthermore, antihypertensive treatment in patients with microalbuminuria has been shown to reduce albumin excretion and prevent the progression to overt proteinuria [4]. However, the temporal relationship between microalbuminuria and rises in BP has not been clarified. Whereas some studies have shown that increases in BP precede microalbuminuria and could influence its development [5, $6]$, other studies have not supported this hypothesis and suggested that the two phenomena could occur together [7, 8] or that BP increases only after the onset of microalbuminuria [9]. A greater understanding of the relationship between $\mathrm{BP}$ and microalbuminuria could be of particular relevance for the early identification of patients at risk of complications and the implementation of preventive and therapeutic strategies. However, most of the studies attempting to explore this relationship have been based on office BP [6,9] and have mainly been conducted in adult populations $[6,8]$. Twenty-four hour ambulatory BP (ABP) monitoring (ABPM) presents several advantages over office BP measurements [10, 11]. In particular, it has a better correlation with target organ damage [12] and is more indicative of cardiovascular morbidity and mortality [12]. It permits evaluation of the circadian rhythm of BP, which may also be of significance $[10,11]$.

Using ABPM, the most common finding in normotensive patients with type 1 diabetes and microalbuminuria has been a blunted decline in BP overnight [13, 14], the socalled 'non-dipping' phenomenon. A high prevalence of non-dippers has also been described in normoalbuminuric patients and related to the future development of microalbuminuria [15]. However, significant overlaps in the fall in BP overnight exist between normoalbuminuric and microalbuminuric patients $[8,16]$. Therefore, the predictive value of the 'non-dipping' phenomenon on future microalbuminuria is not proven.

Microalbuminuria is often preceded by a progressive rise in albumin excretion within the normal range and this has been found to be an important predictor of microalbuminuria and overt diabetic nephropathy [17-21]. Assessing the relationship between albumin excretion and ABP as continuous variables may increase understanding of the role of BP in the pathogenesis of microalbuminuria. We hypothesised that there might be a close relationship between albumin excretion and $\mathrm{ABP}$, even within the normal range, and that ABP might predict the risk of developing microalbuminuria in young people with type 1 diabetes.

\section{Methods}

Study population and study design: the Nephropathy Family Study (NFS) cohort The NFS cohort was set up in 2000 as part of the Juvenile Diabetes Research Foundation/ Wellcome Trust Diabetes Inflammation Laboratory: Genetic Resource Investigating Diabetes study [22]. Between 2000 and 2005, 1,066 patients, aged 10-16 years, who had developed type 1 diabetes before the age of 16 years, were recruited throughout four regions of England (East Anglia, Birmingham, Bristol and Oxford). Patients with insulintreated diabetes secondary to other pathologies were excluded. Similarly, children with chronic renal disease or 
other chronic diseases likely to affect renal function were excluded. The median duration of follow-up is currently 2.6 (interquartile range 1.8-3.7) years.

The longitudinal study schedule comprised annual collection of three consecutive early-morning urine specimens for centralised measurement of albumin:creatinine ratio (ACR) and blood samples for measurements of $\mathrm{HbA}_{1 \mathrm{c}}$. Annual assessments also included measurements of height and weight.

Twenty-four hour ABPM was performed in a subgroup of 509 children from the NFS cohort and assessed in relation to ACR. In addition, 287 of these patients had at least 1 year follow-up after the ABPM was performed, with at least one set of three consecutive early morning urinary samples for the assessment of ACR, a centralised measurement of $\mathrm{HbA}_{1 \mathrm{c}}$ and clinical data, and in these patients the potential associations between baseline $\mathrm{ABP}$ and future changes in ACR were also assessed.

Ethics approval was obtained from the regional ethics committee, with written informed consent from the parents. Children were asked to assent before entering the study.

$A B P M$ ABPM was performed with a portable non-invasive oscillometric recorder (Diasys Integra II, Novacor, France). The validity of this monitor has been previously confirmed [23]. An appropriate cuff size was fitted on the basis of the arm circumference of each patient, and applied on the nondominant arm. ABPM was performed during a normal day with typical activities but patients were asked to avoid vigorous exercises and to keep their arm relaxed during each daytime inflation. A measurement was automatically repeated if values were outside the following intervals of measurement validity: systolic $\mathrm{BP}$ (SBP) $<50 \mathrm{mmHg}$; diastolic BP (DBP) $<30 \mathrm{mmHg}$ or $>150 \mathrm{mmHg}$; heart rate (HR) $<35$ beats per min and $>250$ beats per min; pulse pressure $<10$ and $>150 \mathrm{mmHg}$ at $\mathrm{SBP}<120 \mathrm{mmHg}$ and pulse pressure $<15$ and $>150 \mathrm{mmHg}$ at $\mathrm{SBP}>120 \mathrm{mmHg}$. Furthermore, all patients were asked to record in a diary the time they went to bed and the time they awoke, as well as exercise periods and the quality of the sleep.

$\mathrm{BP}$ readings were obtained at $30 \mathrm{~min}$ intervals during the daytime (07:00-22:00 hours) and at 60 min intervals during the night-time (22:00-07:00 hours). At the end of the ABPM, the monitor data were downloaded to a personal computer equipped with DiasySoft software for the analysis of the measurements. All readings taken during $24 \mathrm{~h}$ were used to calculate mean $24 \mathrm{~h}$ SBP, DBP and HR, whereas mean daytime and night-time SBP, DBP and HR were calculated based on the awake and asleep periods, calculated from diary times. To characterise the circadian BP rhythm, the percentage of nocturnal fall in SBP and DBP was calculated using the formula: [daytime SBP (or DBP)-night-time SBP (or DBP)]/ [daytime SBP (or DBP)] $\times 100$. Patients were classified as dippers if their daytime SBP and/or DBP decreased by at least $10 \%$ during the night; all other patients were classified as non-dippers [24].

Albumin and creatinine measurements All urine samples were assessed centrally in a single reference laboratory at $\mathrm{St}$ Thomas's Hospital, London, UK. Samples were stored at $-70^{\circ} \mathrm{C}$ prior to being analysed. Albumin was measured centrally by a double-antibody ELISA method. The within and in-between assay CV values were $6 \%$ and $12 \%$, respectively. Creatinine was initially measured using a modified Jaffe method (Unimate 7; Roche Diagnostic Systems, Rotkreuz, Switzerland) on a Cobas Mira (Roche Diagnostic Systems) automated spectrophotometer (CV was $2 \%$ at $2.2 \mathrm{mmol} / \mathrm{l}$ ) and, more recently, by stable isotope dilution electrospray mass spectrometry-mass spectrometry, as previously reported [25].

$H b A_{1 c}$ measurements All samples were analysed centrally in Cambridge on a TOSOH G7 analyser (Tosoh Bioscience, Redditch, UK), using HPLC and absorbance change detection, and DCCT aligned methods. The normal range was $4.9-6.3 \%$ and the CV was $4.8 \%$ at a level of $5.5 \%$, and $6.6 \%$ at a level of $10.1 \%$.

Calculations BMI was calculated as weight $/$ height $^{2}$. Standard deviation scores (SDS) for height, weight and BMI were calculated using the British 1990 Growth Reference and Cole's LMS method [26].

The mean of all $\mathrm{HbA}_{1 \mathrm{c}}$ measurements collected from the time of recruitment into the study until the ABPM assessment was calculated.

ACR was summarised as the geometric mean of three consecutive early morning urine samples and $\log _{10}$ transformed for analyses. Microalbuminuria was defined as an ACR between 3.5 and $35 \mathrm{mg} / \mathrm{mmol}$ in male patients and between 4.0 and $47 \mathrm{mg} / \mathrm{mmol}$ in female patients in two out of three consecutive early morning urine collections during an annual assessment (corresponding to an overnight albumin excretion rate of $20-200 \mu \mathrm{g} / \mathrm{min}$ and an ACR of 2.4 and 2.2 $\mathrm{SD}$ above the geometric mean in normal male and female adolescents [27]). Clinical proteinuria was defined as an ACR $>35 \mathrm{mg} / \mathrm{mmol}$ in male patients, and $>47 \mathrm{mg} / \mathrm{mmol}$ in female patients (equivalent to $>200 \mu \mathrm{g} / \mathrm{min}$ ) [27].

The most recent ACR assessment in relation to the time when ABPM was measured (within the previous 6 months) was used for the initial cross-sectional analyses. Subsequently, we calculated a longitudinally adjusted ACR trait, using all available assessments after ABPM was performed. This was calculated as follows: the geometric mean for each assessment was computed and $\log _{10}$ transformed. Then, in order to exclude the effect of significant determinants of ACR, such as duration of diabetes, sex and glycaemic control, we used a 
regression model, where $\log _{10}$ ACR was regressed against these variables and residuals (observed-fitted values) were derived [21]. Finally, the average residual for each patient was calculated and taken as the mean adjusted ACR trait.

BP SDS for $24 \mathrm{~h}$, daytime and night-time SBP and DBP were calculated using the European reference standards [28]. Hypertension was defined as an $\operatorname{SDS}>1.65$, adjusted for sex and height (corresponding to the 95th percentile in a standard normal distribution) [28].

Statistical analyses Data are summarised as means \pm SD or median (interquartile range) unless otherwise specified. Non-normally distributed variables, such as ACR, were $\log _{10}$ transformed before analysis. A one-sample $t$ test was used to compare BP variables, expressed as an SDS, with published normative data (mean $0 \pm \mathrm{SD}$ ). Comparisons between different groups were performed by an unpaired $t$ test. Comparisons across categories were made using $\chi^{2}$ or Fisher's exact test. Association between variables was performed by Pearson correlation analysis, and expressed as correlation coefficient $r$, and by multiple regression analysis, and results are expressed as regression coefficient $B \pm \mathrm{SE}$ or as $\beta$ coefficients. Cox regression hazard regression analysis was performed to assess the proportional contribution of covariates, and in particular of ABPM variables to the risk of developing microalbuminuria during follow-up. $p$ values $<0.05$ were taken as significant. Given that many tests were undertaken, a stricter more conserva- tive $p$ value of $<0.01$ was also applied in order to give a more cautious interpretation.

\section{Results}

General characteristics of the study population and ABPM data ABPM was performed in 509 young patients with type 1 diabetes (278 male and 231 female). The general characteristics of the study population and for male patients and female patients separately are shown in Table 1. Sixty out of the 509 patients had their ACR in the microalbuminuric range at the time when the ABPM was performed or had presented a transient or intermittent microalbuminuria before the ABPM assessment. These patients were included in the analysis of the relationship between ACR and ABP as this allowed us to have a wider range of $\mathrm{ACR}$ to be tested against $\mathrm{ABP}$. However, we excluded these patients for the longitudinal analysis in order not to confound our assessment of the predictive value of $\mathrm{ABP}$ on future development of microalbuminuria.

Table 1 shows mean values for $24 \mathrm{~h}$, and daytime and night-time, SBP, DBP and HR as well as the night-time fall for SBP and DBP. In 23 patients, data for the overnight period were incomplete and therefore only readings during the daytime were included in the analysis. None of the patients was on treatment for hypertension at baseline or during follow-up.
Table 1 General characteristics and ABPM of the study group

Data are means $\pm \mathrm{SD}$, median (interquartile range) or $n(\%)$

${ }^{\text {a }}$ Differences in ABPM variables persisted after adjusting for height and BMI SDS. Data for $24 \mathrm{~h}$ and night-time BP are for 486 patients (266 male and 220 female), with complete ABPM readings

$p$ values are for the comparison between male patients and female patients

\begin{tabular}{lllll}
\hline Characteristics/ABPM & All patients & Male patients & Female patients & $p$ value \\
\hline$n$ & 509 & 278 & 231 & \\
Age at assessment (years) & $15.8 \pm 2.3$ & $15.8 \pm 2.2$ & $15.7 \pm 2.4$ & $\mathrm{NS}$ \\
Age at diagnosis (years) & $8.9 \pm 3.8$ & $9.0 \pm 3.9$ & $8.8 \pm 3.6$ & $\mathrm{NS}$ \\
Duration (years) & $6.9 \pm 3.8$ & $6.8 \pm 3.9$ & $7.0 \pm 3.7$ & $\mathrm{NS}$ \\
HbA $_{1 \mathrm{c}}$ (\%) & $9.3 \pm 1.6$ & $9.1 \pm 1.6$ & $9.4 \pm 1.7$ & $\mathrm{NS}$ \\
Insulin (U kg $^{-1}$ day $^{-1}$ ) & $1.1 \pm 0.3$ & $1.1 \pm 0.3$ & $1.1 \pm 0.4$ & $\mathrm{NS}$ \\
Height SDS & $0.15 \pm 1.04$ & $0.06 \pm 1.05$ & $0.26 \pm 1.01$ & 0.03 \\
BMI SDS & $0.97 \pm 1.15$ & $0.85 \pm 1.13$ & $1.10 \pm 1.16$ & 0.03 \\
ACR (mg/mmol) & $0.88(0.63-1.42)$ & $0.78(0.78-1.17)$ & $1.00(0.67-1.80)$ & $<0.001$ \\
ABPM & & & & $<0.001$ \\
24 h SBP (mmHg) & $114 \pm 10$ & $116 \pm 10$ & $112 \pm 10$ & $\mathrm{NS}$ \\
24 h DBP (mmHg) & $72 \pm 7$ & $72 \pm 7$ & $72 \pm 7$ & $<0.001$ \\
24 h HR (beats per min) & $83 \pm 11$ & $81 \pm 10$ & $86 \pm 11$ & $<0.001$ \\
Day SBP (mmHg) & $117 \pm 11$ & $119 \pm 11$ & $115 \pm 10$ & $\mathrm{NS}$ \\
Day DBP (mmHg) & $74 \pm 8$ & $74 \pm 8$ & $74 \pm 7$ & $<0.001$ \\
Day HR (beats per min) & $88 \pm 12$ & $85 \pm 11$ & $90 \pm 12$ & 0.001 \\
Night SBP (mmHg) & $105 \pm 11$ & $106 \pm 11$ & $103 \pm 10$ & 0.03 \\
Night DBP (mmHg) & $67 \pm 8$ & $68 \pm 8$ & $66 \pm 8$ & $<0.001$ \\
Night HR (beats per min) & $70 \pm 10$ & $68 \pm 10$ & $112(50.9)$ & $\mathrm{NS}$ \\
Non-dippers SBP (\%) & $235(48.4)$ & $123(46.2)$ & $120(54.5)$ & 0.03 \\
Non-dippers DBP (\%) & $292(60.1)$ & $172(64.7)$ & & \\
\hline & & & \\
\end{tabular}


The evaluation of the BP SDS showed values higher than those in the reference population for DBP, particularly at night (mean \pm SD; 24 h DBP: $0.78 \pm 1.24$; daytime DBP: $0.20 \pm 1.33$; night-time DBP: $1.74 \pm 1.19$; all $p<0.01$ ), but lower than the reference values for SBP ( $24 \mathrm{~h}$ SBP: $-0.18 \pm$ 1.34 , daytime SBP: $-0.43 \pm 1.38 ; p<0.01)$. No differences were found for night-time $\operatorname{SBP}(0.10 \pm 1.27, p>0.05)$. The prevalence of hypertension was as follows: $24 \mathrm{~h}$ SBP: $8.6 \%$; 24 h DBP: 24.4\%; daytime SBP: 5.7\%; daytime DBP: 12.9\%; night-time SBP: $9.4 \%$; night-time DBP: $48.8 \%$. The prevalence of abnormal BP values was not different in male when compared with female patients (all $p>0.05$ ).

Relationship between ABP and other variables All potential simple correlations between $\mathrm{ABP}$ and age, duration of diabetes, $\mathrm{HbA}_{1 \mathrm{c}}, \log _{10} \mathrm{ACR}$, insulin dose, height SDS and BMI SDS were analysed (Electronic supplementary material [ESM] Table 1). Significant correlations were found between $\log$ ACR at the time of ABPM assessment and all ABPM variables (all $p<0.001$ ). $\mathrm{HbA} 1_{\mathrm{c}}$ was significantly related to DBP $(p<0.01)$ but not to SBP. Insulin dose was significantly related to $24 \mathrm{~h}$ and daytime SBP and night-time DBP $(p<$ $0.05)$. BMI SDS was significantly related to all ABPM variables $(p \leq 0.01)$. Duration of diabetes was only related to $24 \mathrm{~h}$ and daytime DBP $(p<0.01)$. All ABPM variables were significantly related to age at assessment $(p<0.01)$.

Relationship between $A C R$ and $A B P$ In multivariate analysis, $\log _{10}$ ACR was independently related to all ABP variables $(B \pm \mathrm{SE} ; 24 \mathrm{~h}$ SBP: $7.5 \pm 1.4 ; 24 \mathrm{~h}$ DBP: $4.5 \pm 1.0$; daytime SBP: $8.0 \pm 1.5$; daytime DBP: $4.7 \pm 1.1$; night-time SBP: $7.9 \pm 1.5$; night-time DBP: $4.1 \pm 1.1$; all $p<0.001$ ), after adjustment for potential confounders, such as age, sex, BMI SDS and $\mathrm{HbA}_{1 \mathrm{c}}$.

Future microalbuminuric vs normoalbuminuric patients After the ABPM evaluation, 287 patients were followed for at least 1 year (follow-up: median [range]: 2.2 [1.0-5.5] years). The baseline characteristics of these patients were similar to those who did not have follow-up assessments, apart from a slightly younger age $(15.3 \pm 2.1$ vs $16.3 \pm$ 2.3 years), a shorter duration of diabetes ( $6.2 \pm 3.6$ vs $7.8 \pm$ 3.9 years) and lower mean $\mathrm{HbA}_{1 \mathrm{c}}$ levels $(9.0 \pm 1.5$ vs $9.6 \pm 1.7 \%$ ), but there were no differences in $\mathrm{ABPM}$ variables.

Daytime SBP $(\beta=0.15, p=0.01)$ and DBP $(\beta=0.21, p<$ $0.001)$ and night-time SBP $(\beta=0.18, p=0.03)$ were significantly related to the adjusted mean ACR values during follow-up (Fig. 1). During this period, out of the 250 patients who were normoalbuminuric at baseline, 19 developed microalbuminuria and they were defined as the 'future microalbuminuric' group. For the 'future microalbuminuric' group median time to onset of microalbuminuria after the ABPM assessment was $2.0(0.2-4.0)$ years. A comparison was performed between these 19 'future microalbuminuric' patients and the remaining 231 patients who remained normoalbuminuric during followup (Table 2). Significant differences between the two groups were found for baseline $\mathrm{HbA}_{1 \mathrm{c}}$ and $\mathrm{ACR}$, both being higher in the 'future microalbuminuric' group $(p<0.001)$. 'Future microalbuminuric' patients had higher $24 \mathrm{~h}$, daytime and night-time DBP than normoalbuminuric patients, but there was no difference in the nocturnal fall in BP between the two groups (Table 2). The differences in $24 \mathrm{~h}$ and daytime DBP between the groups persisted also after adjustment for $\mathrm{HbA}_{1 \mathrm{c}}$ and $\log _{10}$ ACR (Table 2).

Cox proportional hazard regression In a Cox proportional regression analysis with the development of microalbuminuria as the outcome variable and years of follow-up after the ABPM assessment as the time variable, for each $1 \mathrm{mmHg}$ increase in daytime DBP, the risk of developing microalbuminuria was 1.11 (95\% CI 1.03-1.19), $p=0.005$ (Table 3). In this model, $\mathrm{HbA}_{1 \mathrm{c}}$ and baseline ACR also significantly contributed to the risk of developing microalbuminuria, whereas there was no statistically significant effect of sex, age at diagnosis or duration of diabetes (Table 3). The association between daytime DBP and microalbuminuria persisted also after adjusting for the other ABPM variables $(p=0.002)$.

In contrast, when the same analysis was repeated separately for each of the other ABPM variables, there was no statistically significant contribution of them to the risk of developing microalbuminuria.

Dippers vs non-dippers Two hundred and thirty-five (48\%) patients were non-dippers for SBP and 292 (60\%) were non-dippers for DBP. A comparison between dippers and non-dippers is shown in Table 4.

Dippers and non-dippers were comparable for age, duration of diabetes and BMI SDS. SBP non-dippers had higher $\mathrm{HbA}_{1 \mathrm{c}}$ values when compared with dippers. No differences in $\log _{10}$ ACR were found between dippers and non-dippers, whereas HR variables were significantly different between the two groups.

The fall in SBP and DBP overnight was not related to age, duration of diabetes, $\mathrm{HbA}_{1 \mathrm{c}}, \log _{10} \mathrm{ACR}$, insulin dose, height SDS and BMI SDS (all $p>0.05$ ). In contrast, it was related to 24 h HR (SBP fall: $r=0.12, p=0.01$; DBP fall: $r=$ $0.15, p<0.01$ ) and daytime HR (SBP fall: $r=0.18, p<0.01$; DBP fall: $r=0.20, p<0.01)$. In addition, there was no contribution of the nocturnal fall in BP to the risk of developing microalbuminuria in the Cox model $(p>0.05)$.

\section{Discussion}

In this population of young people with type 1 diabetes we found a high prevalence of abnormal BP values, particu- 

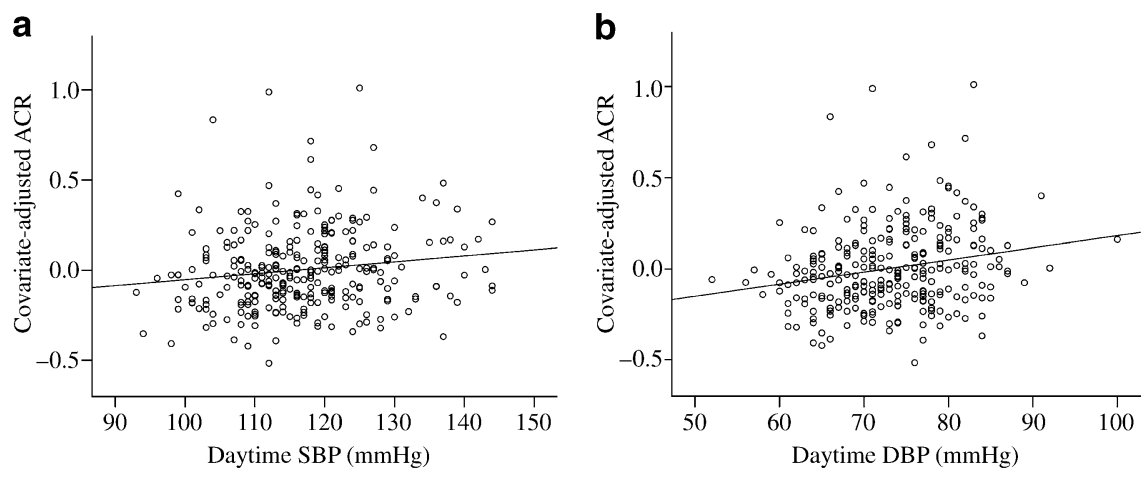

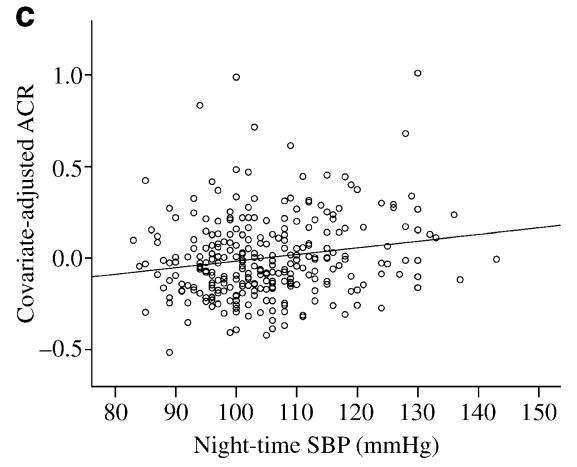

Fig. 1 Relationship between baseline ABPM variables and mean adjusted ACR ( $\log _{10}$ scale) during follow-up. ACR was adjusted for duration of diabetes, sex and $\mathrm{HbA}_{1 \mathrm{c}}$, by calculating residuals, which were then plotted against ABP. Daytime SBP: $\beta=0.15$ (a); daytime DBP: $\beta=0.21$ (b); night-time SBP: $\beta=0.18$ (c)

progression to microalbuminuria. This supports our hypothesis that rises in ACR, even within the normal range, may parallel similar progressive increases in ABP. The associations between ACR and ABP at baseline were not explained by $\mathrm{HbA}_{1 \mathrm{c}}$ or $\mathrm{BMI}$, although both these factors were independent contributors of $\mathrm{ABP}$, in line with the findings of previous studies [24, 30, 31]. Similarly, SBP and DBP were independently associated with future changes in ACR during the subsequent follow-up period. In addition, patients developing microalbuminuria during up, and daytime DBP was independently associated with

Table 2 General characteristics and ABPM variables of normoalbuminuric and future microalbuminuric patients

\begin{tabular}{|c|c|c|c|c|}
\hline Characteristic/variable & Normoalbuminuric $(n=231)$ & Future microalbuminuric $(n=19)$ & $p$ value & Adjusted $p$ value ${ }^{\mathrm{a}}$ \\
\hline $\operatorname{Sex}(M / F)$ & $134 / 97$ & $8 / 11$ & NS & \\
\hline Age (years) & $15.2 \pm 2.1$ & $15.5 \pm 2.2$ & NS & \\
\hline Age at diagnosis (years) & $9.2 \pm 3.8$ & $9.0 \pm 4.1$ & NS & \\
\hline Duration (years) & $6.1 \pm 3.6$ & $6.6 \pm 4.0$ & NS & \\
\hline BMI SDS & $0.94 \pm 1.14$ & $0.72 \pm 1.07$ & NS & \\
\hline $\mathrm{HbA}_{1 \mathrm{c}}(\%)$ & $8.8 \pm 1.4$ & $9.9 \pm 1.7$ & 0.001 & \\
\hline $\mathrm{ACR}(\mathrm{mg} / \mathrm{mmol})$ & $0.78(0.59-1.13)$ & $1.23(0.97-2.06)$ & $<0.001$ & \\
\hline ACR at last visit (mg/mmol) & $0.82(0.59-1.22)$ & $5.70(5.05-7.51)$ & $<0.001$ & \\
\hline \multicolumn{5}{|l|}{$\mathrm{ABP}$ and heart rate } \\
\hline $24 \mathrm{~h} \mathrm{SBP}(\mathrm{mmHg})$ & $113 \pm 10$ & $115 \pm 8$ & NS & NS \\
\hline 24 h DBP $(\mathrm{mmHg})$ & $71 \pm 7$ & $76 \pm 6$ & 0.001 & 0.03 \\
\hline 24 h HR (beats per min) & $82 \pm 11$ & $87 \pm 7$ & 0.02 & NS \\
\hline Day SBP (mmHg) & $116 \pm 10$ & $119 \pm 8$ & NS & NS \\
\hline Day DBP (mmHg) & $72 \pm 7$ & $79 \pm 6$ & $<0.001$ & 0.003 \\
\hline Day HR (beats per min) & $86 \pm 12$ & $93 \pm 7$ & 0.01 & NS \\
\hline Night SBP (mmHg) & $104 \pm 10$ & $107 \pm 14$ & NS & NS \\
\hline Night DBP (mmHg) & $66 \pm 7$ & $70 \pm 10$ & 0.03 & NS \\
\hline Night HR (beats per min) & $69 \pm 10$ & $75 \pm 15$ & 0.02 & NS \\
\hline SBP fall $(\%)$ & $10.4 \pm 7.6$ & $9.8 \pm 10.2$ & NS & NS \\
\hline DBP fall $(\%)$ & $7.8 \pm 8.7$ & $11.4 \pm 11.5$ & NS & NS \\
\hline
\end{tabular}

Data are means $\pm \mathrm{SD}$, median (interquartile range) or $\%$

${ }^{\text {a }}$ Adjusted for $\mathrm{HbA}_{1 \mathrm{c}}$ and $\mathrm{ACR}$ 
Table 3 Cox proportional hazard regression for the development of microalbuminuria

\begin{tabular}{lllr}
\hline Variable & $\operatorname{Exp}(\mathrm{B})^{\mathrm{a}}$ & $95 \% \mathrm{CI}$ & $p$ value \\
\hline Day DBP (mmHg) & 1.11 & $1.03-1.19$ & 0.005 \\
$\mathrm{HbA}_{1 \mathrm{c}}(\%)$ & 1.44 & $1.08-1.92$ & 0.014 \\
$\mathrm{ACR}(\mathrm{mg} / \mathrm{mmol})$ & 2.99 & $1.82-4.90$ & $<0.001$
\end{tabular}

Time variable is years of follow-up after the ABPM assessment

${ }^{\mathrm{a}} \operatorname{Exp}(\mathrm{B})$ is for 1 -unit increase in the continuous predictor. Other covariates included in the model, but not statistically significant were: age at diagnosis, sex and duration of diabetes

follow-up had higher daytime DBP at the baseline ABPM assessment compared with patients who remained normoalbuminuric. After adjusting for potential confounders, a $1 \mathrm{mmHg}$ rise in daytime DBP increased the risk of developing microalbuminuria by about $11 \%$. This significant effect of daytime DBP persisted even after adjusting for the other ABPM variables, therefore strengthening its independent and dominant association with the progression to microalbuminuria. In adults, pulse pressure has been found to be a key determinant of cardiovascular events and microvascular complications of diabetes [32-34]. In contrast, we could not find any additional or independent effect of pulse pressure over DBP on urinary albumin excretion and on the progression to microalbuminuria during followup (data not shown). However, this different finding could be related to the younger age of our study participants when compared with that of other populations assessed in previous studies [33].

The results of the present study are in line with previous reports, showing that both SBP and DBP are associated with albumin excretion $[16,35]$ as well as with changes in renal function, such as a decreased renal plasma flow and GFR [16].

Our findings are also not dissimilar from those reported by Lurbe et al. [15] in a population of 75 adolescents with type 1 diabetes. In fact, in that study, the most relevant finding at the first ABPM measurement, in patients who later manifested microalbuminuria, was a similar increase in daytime DBP. Only 2 years later, when they performed a second ABPM, a marked increase in nocturnal BP, with a blunted night-time BP fall was detected. Increases in DBP during daytime might therefore be one of the first BP alterations in patients at risk of developing microalbuminuria, and appears to represent an important variable to monitor over time.

In our population we also assessed the 'non-dipping' phenomenon and found a high prevalence of non-dippers, particularly for DBP. A blunted diurnal BP rhythm has often been reported in patients with type 1 diabetes, with a worsening of this abnormal pattern with progression from

Table 4 Dippers vs non-dippers for SBP and DBP

\begin{tabular}{|c|c|c|c|c|c|c|}
\hline Characteristic/variable & $\begin{array}{l}\text { SBP } \\
\text { Dippers }\end{array}$ & Non-dippers & $\begin{array}{l}\mathrm{DBP} \\
p \text { value }\end{array}$ & Dippers & Non-dippers & $p$ value \\
\hline$n(\%)$ & $251(52)$ & $235(48)$ & & $194(40)$ & $292(60)$ & \\
\hline Age (years) & $15.7 \pm 2.3$ & $15.8 \pm 2.3$ & NS & $15.8 \pm 2.4$ & $15.8 \pm 2.2$ & NS \\
\hline Age at diagnosis (years) & $8.8 \pm 3.9$ & $9.0 \pm 3.7$ & NS & $8.6 \pm 3.8$ & $9.1 \pm 3.8$ & NS \\
\hline Duration (years) & $6.9 \pm 3.9$ & $6.8 \pm 3.8$ & NS & $7.1 \pm 3.9$ & $6.7 \pm 3.8$ & NS \\
\hline $\mathrm{HbA}_{1 \mathrm{c}}(\%)$ & $9.1 \pm 1.6$ & $9.5 \pm 1.7$ & 0.02 & $9.3 \pm 1.7$ & $9.4 \pm 1.7$ & NS \\
\hline Insulin $\left(\mathrm{U} \mathrm{kg}^{-1}\right.$ day $\left.^{-1}\right)$ & $1.1 \pm 0.3$ & $1.1 \pm 0.3$ & NS & $1.1 \pm 0.3$ & $1.1 \pm 0.4$ & NS \\
\hline Height SDS & $0.26 \pm 1.10$ & $0.06 \pm 1.04$ & 0.04 & $0.23 \pm 1.07$ & $0.13 \pm 1.04$ & NS \\
\hline BMI SDS & $0.8 \pm 0.9$ & $0.7 \pm 1.0$ & NS & $0.8 \pm 1.0$ & $0.8 \pm 0.9$ & NS \\
\hline ACR (mg/mmol) & $0.85(0.62-1.24)$ & $0.92(0.62-1.65)$ & NS & $0.93(0.61-1.40)$ & $0.83(0.63-1.42)$ & NS \\
\hline \multicolumn{7}{|l|}{$\mathrm{ABP}$ and heart rate } \\
\hline 24 h SBP (mmHg) & $115 \pm 10$ & $112 \pm 10$ & 0.01 & $115 \pm 11$ & $113 \pm 9$ & NS \\
\hline 24 h DBP (mmHg) & $72 \pm 7$ & $72 \pm 7$ & NS & $73 \pm 7$ & $71 \pm 7$ & 0.001 \\
\hline $24 \mathrm{~h} \mathrm{HR}$ (beats per min) & $83 \pm 11$ & $82 \pm 10$ & NS & $84 \pm 11$ & $82 \pm 10$ & 0.004 \\
\hline Day SBP (mmHg) & $120 \pm 11$ & $113 \pm 10$ & $<0.001$ & $119 \pm 12$ & $115 \pm 10$ & 0.001 \\
\hline Day DBP (mmHg) & $74 \pm 8$ & $73 \pm 7$ & 0.02 & $77 \pm 8$ & $71 \pm 7$ & $<0.001$ \\
\hline Day HR (beats per min) & $88 \pm 12$ & $86 \pm 11$ & 0.04 & $89 \pm 12$ & $86 \pm 11$ & $<0.001$ \\
\hline Night SBP (mmHg) & $101 \pm 9$ & $109 \pm 11$ & $<0.001$ & $103 \pm 11$ & $106 \pm 10$ & 0.009 \\
\hline Night DBP (mmHg) & $66 \pm 7$ & $68 \pm 8$ & 0.001 & $63 \pm 7$ & $69 \pm 7$ & $<0.001$ \\
\hline Night HR (beats per min) & $69 \pm 9$ & $71 \pm 11$ & 0.03 & $70 \pm 10$ & $70 \pm 10$ & NS \\
\hline
\end{tabular}

Data are means $\pm \mathrm{SD}$, median (interquartile range) or $n(\%)$ 
microalbuminuria to macroalbuminuria [13]. In type 1 diabetes, autonomic dysfunction, reflected by sympathetic overactivity, and alterations in volume regulation have been suggested as potential mechanisms implicated in the pathogenesis of the blunted diurnal rhythm in BP [36-38]. In line with a previous study [39], we found that the most important factors associated with the nocturnal fall in BP were the HR variables, suggesting a role of autonomic dysfunction.

The 'non-dipping' phenomenon is a condition known to be associated in adults with an increased risk of end-organ damage $[12,40,41]$ and this finding has generated a lot of interest for the potential relationship with vascular disease. Therefore, the high prevalence of impaired fall in nocturnal BP we found may be clinically significant and be associated with an increased risk for the development and/ or progression of microvascular and macrovascular complications. However, we could not find any relationship between albumin excretion and the nocturnal fall in BP. This might be related to the high variability in the BP fall overnight in our study population and therefore to significant overlaps across the range of ACR. This high variability associated with the small number of 'future microalbuminuric' patients could have also reduced the power of our study to detect a difference in the nocturnal fall between the normoalbuminuric and 'future microalbuminuric' groups. In addition, in the present study, ABPM was performed only once, and therefore we could not assess the reproducibility of the dipping/non-dipping, which appears to be variable [42-44] and lower than that of the absolute daytime and night-time values [42], and this could have influenced the study results.

However, previous reports have highlighted a high percentage of non-dippers already among normoalbuminuric populations with type 1 diabetes [45], wide overlaps between normoalbuminuric and microalbuminuric patients $[8,16]$ as well as lack of associations between the nocturnal dipping and microalbuminuria after adjusting for potential confounders [8]. Based on these data and those from our study, the 'non-dipping' phenomenon does not appear to be predictive of risk of microalbuminuria, but it cannot be excluded that it may still be an important determinant of the rate of progression from microalbuminuria to overt diabetic nephropathy. Future studies, with repeated ABPM assessments and longer term follow-up could help in clarifying this aspect.

In conclusion, in this cohort of young people with type 1 diabetes, $\mathrm{ABP}$ was significantly related to $\mathrm{ACR}$, suggesting that increasing albumin excretion, even in the normoalbuminuric range, may be associated with parallel rises in arterial BP. In addition, daytime DBP was independently associated with progression to microalbuminuria during follow-up.

Detecting these alterations in BP could represent an important means for an early identification of patients at risk, before the clinical manifestation of microalbuminuria, and therefore prompt early implementation of preventive measures.

Acknowledgements The Nephropathy Family Study (NFS) is funded by The Juvenile Diabetes Research Foundation (JDRF) and the Wellcome Trust. We acknowledge the study field workers, paediatricians, physicians and diabetes nurse specialists involved in the NFS, JDRF, Wellcome Trust, National Institute for Health Research (NIHR) Cambridge Comprehensive Biomedical Research Centre and Novacor UK. We thank K. L. Ng (United Christian Hospital, Hong Kong) for his help with the BP data analysis. In addition, this study was supported by a European Society for Paediatric Endocrinology (ESPE) Research Fellowship, sponsored by Novo Nordisk A/S (to M. L. Marcovecchio). The results of this study were presented at the 47th ESPE annual meeting 2008, Istanbul, Turkey.

Duality of interest The authors declare that there is no duality of interest associated with this manuscript.

\section{References}

1. Gross JL, de Azevedo MJ, Silveiro SP, Canani LH, Caramori ML, Zelmanovitz T (2005) Diabetic nephropathy: diagnosis, prevention, and treatment. Diabetes Care 28:164-176

2. Rossing P, Hougaard P, Borch-Johnsen K, Parving HH (1996) Predictors of mortality in insulin dependent diabetes: 10 year observational follow up study. BMJ 313:779-784

3. Rudberg S, Stattin EL, Dahlquist G (1998) Familial and perinatal risk factors for micro- and macroalbuminuria in young IDDM patients. Diabetes 47:1121-1126

4. ACE Inhibitors in Diabetic Nephropathy Trialist Group (2001) Should all patients with type 1 diabetes mellitus and microalbuminuria receive angiotensin-converting enzyme inhibitors? A meta-analysis of individual patient data. Ann Intern Med 134:370-379

5. Moore WV, Donaldson DL, Chonko AM, Ideus P, Wiegmann TB (1992) Ambulatory blood pressure in type I diabetes mellitus. Comparison to presence of incipient nephropathy in adolescents and young adults. Diabetes 41:1035-1041

6. Anon (1993) Risk factors for development of microalbuminuria in insulin dependent diabetic patients: a cohort study. Microalbuminuria Collaborative Study Group, United Kingdom. BMJ 306:1235-1239

7. Schultz CJ, Neil HA, Dalton RN, Konopelska Bahu T, Dunger DB (2001) Blood pressure does not rise before the onset of microalbuminuria in children followed from diagnosis of type 1 diabetes. Oxford Regional Prospective Study Group. Diabetes Care 24:555560

8. Poulsen PL, Hansen KW, Mogensen CE (1994) Ambulatory blood pressure in the transition from normo- to microalbuminuria. A longitudinal study in IDDM patients. Diabetes 43:1248-1253

9. Mathiesen ER, Ronn B, Jensen T, Storm B, Deckert T (1990) Relationship between blood pressure and urinary albumin excretion in development of microalbuminuria. Diabetes 39:245-249

10. Pickering TG, Shimbo D, Haas D (2006) Ambulatory bloodpressure monitoring. N Engl J Med 354:2368-2374

11. Lurbe E, Sorof JM, Daniels SR (2004) Clinical and research aspects of ambulatory blood pressure monitoring in children. $\mathrm{J}$ Pediatr 144:7-16

12. Mancia G, Parati G (2000) Ambulatory blood pressure monitoring and organ damage. Hypertension 36:894-900 
13. Hansen KW, Mau Pedersen M, Marshall SM, Christiansen JS, Mogensen CE (1992) Circadian variation of blood pressure in patients with diabetic nephropathy. Diabetologia 35:1074-1079

14. Lurbe A, Redon J, Pascual JM, Tacons J, Alvarez V, Batlle DC (1993) Altered blood pressure during sleep in normotensive subjects with type I diabetes. Hypertension 21:227-235

15. Lurbe E, Redon J, Kesani A et al (2002) Increase in nocturnal blood pressure and progression to microalbuminuria in type 1 diabetes. N Engl J Med 347:797-805

16. Poulsen PL, Juhl B, Ebbehoj E, Klein F, Christiansen C, Mogensen CE (1997) Elevated ambulatory blood pressure in microalbuminuric IDDM patients is inversely associated with renal plasma flow. A compensatory mechanism? Diabetes Care 20:429-432

17. Hovind P, Tarnow L, Rossing P et al (2004) Predictors for the development of microalbuminuria and macroalbuminuria in patients with type 1 diabetes: inception cohort study. BMJ 328:1105

18. Messent JW, Elliott TG, Hill RD, Jarrett RJ, Keen H, Viberti GC (1992) Prognostic significance of microalbuminuria in insulindependent diabetes mellitus: a twenty-three year follow-up study. Kidney Int 41:836-839

19. Klausen K, Borch-Johnsen K, Feldt-Rasmussen B et al (2004) Very low levels of microalbuminuria are associated with increased risk of coronary heart disease and death independently of renal function, hypertension, and diabetes. Circulation 110:32-35

20. Giorgino F, Laviola L, Cavallo Perin P, Solnica B, Fuller J, Chaturvedi N (2004) Factors associated with progression to macroalbuminuria in microalbuminuric Type 1 diabetic patients: the EURODIAB Prospective Complications Study. Diabetologia 47:1020-1028

21. Dunger DB, Schwarze CP, Cooper JD et al (2007) Can we identify adolescents at high risk for nephropathy before the development of microalbuminuria? Diabet Med 24:131-136

22. UK Children's Diabetes Research: UK GRID: Genetic Resource Investigating Diabetes. Available from www.childhood-diabetes. org.uk/grid.shtml, accessed 6 December 2008

23. O'Brien E, Coats A, Owens P et al (2000) Use and interpretation of ambulatory blood pressure monitoring: recommendations of the British hypertension society. BMJ 320:1128-1134

24. Dost A, Klinkert C, Kapellen T et al (2008) Arterial hypertension determined by ambulatory blood pressure profiles: contribution to microalbuminuria risk in a multicenter investigation in 2105 children and adolescents with diabetes mellitus type 1. Diabetes Care 31:720-725

25. Williams RM, Yuen K, White D et al (2006) Short-term suppression of elevated growth hormone concentrations following insulin-like growth factor 1 administration in young adults with type 1 diabetes does not alter glomerular filtration or albumin excretion rates. Clin Endocrinol (Oxf) 65:439-445

26. Cole TJ (1990) The LMS method for constructing normalized growth standards. Eur J Clin Nutr 44:45-60

27. Schultz CJ, Konopelska-Bahu T, Dalton RN et al (1999) Microalbuminuria prevalence varies with age, sex, and puberty in children with type 1 diabetes followed from diagnosis in a longitudinal study. Oxford Regional Prospective Study Group. Diabetes Care 22:495-502

28. Wuhl E, Witte K, Soergel M, Mehls O, Schaefer F (2002) Distribution of 24-h ambulatory blood pressure in children: normalized reference values and role of body dimensions. J Hypertens 20:1995-2007
29. Shankar RR, Eckert GJ, Saha C, Tu W, Pratt JH (2005) The change in blood pressure during pubertal growth. J Clin Endocrinol Metab 90:163-167

30. Torchinsky MY, Gomez R, Rao J, Vargas A, Mercante DE, Chalew SA (2004) Poor glycemic control is associated with increased diastolic blood pressure and heart rate in children with Type 1 diabetes. J Diabetes Complications 18:220-223

31. Darcan S, Goksen D, Mir S et al (2006) Alterations of blood pressure in type 1 diabetic children and adolescents. Pediatr Nephrol 21:672-676

32. Palmas W, Moran A, Pickering T et al (2006) Ambulatory pulse pressure and progression of urinary albumin excretion in older patients with type 2 diabetes mellitus. Hypertension 48:301-308

33. Franklin SS, Larson MG, Khan SA et al (2001) Does the relation of blood pressure to coronary heart disease risk change with aging? The Framingham Heart Study. Circulation 103:1245-1249

34. Knudsen ST Laugesen E, Hansen KW, Bek T, Mogensen CE, Poulsen PL (2009) Ambulatory pulse pressure, decreased nocturnal blood pressure reduction and progression of nephropathy in type 2 diabetic patients. Diabetologia 52:698-704. doi:10.1007/ s00125-009-1262-6

35. Gallego PH, Gilbey AJ, Grant MT et al (2005) Early changes in 24-hour ambulatory blood pressure are associated with high normal albumin excretion rate in children with type 1 diabetes mellitus. J Pediatr Endocrinol Metab 18:879-885

36. Lafferty AR, Werther GA, Clarke CF (2000) Ambulatory blood pressure, microalbuminuria, and autonomic neuropathy in adolescents with type 1 diabetes. Diabetes Care 23:533-538

37. Poulsen PL, Ebbehoj E, Hansen KW, Mogensen CE (1997) 24-h blood pressure and autonomic function is related to albumin excretion within the normoalbuminuric range in IDDM patients. Diabetologia 40:718-725

38. Hommel E, Mathiesen ER, Giese J, Nielsen MD, Schutten HJ, Parving HH (1989) On the pathogenesis of arterial blood pressure elevation early in the course of diabetic nephropathy. Scand J Clin Lab Invest 49:537-544

39. Karavanaki K, Kazianis G, Kakleas K, Konstantopoulos I, Karayianni C (2007) QT interval prolongation in association with impaired circadian variation of blood pressure and heart rate in adolescents with Type 1 diabetes. Diabet Med 24:1247-1253

40. White WB (2000) Ambulatory blood pressure monitoring: dippers compared with non-dippers. Blood Press Monit 5(Suppl 1):S17S23

41. Izzedine H, Launay-Vacher V, Deray G (2006) Abnormal blood pressure circadian rhythm: a target organ damage? Int J Cardiol 107:343-349

42. Wang X, Poole JC, Treiber FA, Harshfield GA, Hanevold CD, Snieder H (2006) Ethnic and gender differences in ambulatory blood pressure trajectories: results from a 15-year longitudinal study in youth and young adults. Circulation 114:2780-2787

43. Mochizuki Y, Okutani M, Donfeng Y et al (1998) Limited reproducibility of circadian variation in blood pressure dippers and nondippers. Am J Hypertens 11:403-409

44. Delaney A, Pellizzari M, Speiser PW, Frank GR (2009) Pitfalls in the measurement of the nocturnal blood pressure dip in adolescents with type 1 diabetes. Diabetes Care 32:165-168

45. Cohen CN, Filho FM, de Fatima Goncalves M, de Brito Gomes M (2001) Early alterations of blood pressure in normotensive and normoalbuminuric type 1 diabetic patients. Diabetes Res Clin Pract 53:85-90 